\title{
Chronic Lupus Erythematosus and Systemic Scleroderma Coexistent at the Same Patient
}

\author{
Rosa Gimenez-Garcia*, Donna Zhan, Diego Gonzalez-Gonzalez, Daniel Colinas-Reyero \\ Hospital Rio Hortega. Valladolid. Spain
}

*Corresponding Author: Rosa Gimenez-Garcia, Hospital Rio Hortega, Valladolid. Spain, Email: rosagim@hotmail.com

\section{INTRODUCTION}

The term "Overlap syndrome" is referred to the coexistence of clinical manifestations and immunologic characteristics of two or more connective tissue diseases in one patient. The identification of overlap syndrome is important for the prognosis and management of the patient, since the coexistence of two different diseases changes the treatment.

The most frequent diseases associated with the overlap syndrome are lupus, sclerosis, poliomyositis and rheumatoid arthritis. There have been reported several cases of overlap between lupus erythematous and scleroderma, showing predilection for younger women. We present the case of a patient with clinical and histopathologic findings compatible with both lupus erythematosus discoid and systemic scleroderma. [1-2]

\section{CASe Report}

A 50-year-old patient with family history of a daughter with lupus nephritis, was diagnosed of chronic lupus at the age of 30 by biopsy of facial skin lesions. In addition to $\mathrm{HCV}$ phenotype $1 \mathrm{~b}$ chronic liver disease with significant fibrosis.

On initial evaluation, several inflammatory alopecia plaques were seen on the scalp, lesions on the palate and telangiectasia on both palms of the hands. Biopsy of scalp lesions was performed with a result consistent with chronic lupus. Pathological capillaroscopy compatible with Raynaud in active phase. Analytically, there were positive antinuclear antibodies (titer 1:640; anticetromere pattern), antibodies to anticardiolipin and negative anti RNP antibodies, normal blood count and biochemistry except for hypertriglyceridemia. Normal urine test.
The biopsy of scalp lesions revealed areas of vacuolization of the basal layer, observing in the underlying dermis a chronic periadnexal inflammatory infiltrate and mucin. In the pulmonary CT scan areas of subpleural parenchymal destruction (honeycombing) were seen in both lower lobes and a nodule in the lower right lobe.

The patient is in treatment with antimalarial of synthesis, vasodilators and azathioprine.

\section{DISCUSSION}

Connective tissue diseases are autoimmune disorders that manifest with a large range of different clinical features, affecting not only the skin but the whole body. They are classified according to established criteria in which clinical characteristics and specific serological markers are considered. When the patients present several features that cannot be assigned to a single disease they are classified as undifferentiated connective tissue disease. However, it may also be the case that the patient presents well-defined manifestations of two or more connective diseases, this being known as overlap syndrome. The connective tissue diseases that overlap with scleroderma (localized and systemic) are systemic lupus erythematosus, polimyositis, dermatomyositis, rheumatoid arthritis, systemic vasculitis and multiple sclerosis. [3-4]

In lupus erythematosus there may be diffuse alopecia as non-cicatricial alopecia, coinciding with outbreaks of systemic lupus erythematosus, telogen effluvium or alopecia secondary to treatment with antimalarials, corticoids or azathioprine. Cicatricial alopecia is commonly associated with chronic lupus erythematous, and the diagnose is based on the clinic (rounded or oval alopecia plaques, well delimited with 
erythematous and desquamative surface with folicular plugs), the response to the treatment and the distinctive histopathological findings, which includes epidermal atrophy and follicular plugging, an interface dermatitis, a wellcircumscribed lymphocytic infiltration in perivascular and periadnexal disposition, and an abundant deposit of mucin in the superficial and deep dermis. In the trichoscopy the most characteristic findings are the follicular red dots, arborizing telangiectasia and follicular keratotic plugs. [5-6]

In our case, the patient was initially diagnose with chronic lupus, with the appearance of cicatricial alopecia she was derived to the dermatologist where lesions compatible with a CREST syndrome were observed, suggesting an overlap of the two diseases. The clinical feature that indicated the possibility of suffering scleroderma was the presence of Raynaud phenomenon observed through the capillaroscopy. To detect visceral involvement a pulmonary CT scan was made, showing a honeycombing pattern in both lower lobes, a common form in which scleroderma affects the lungs[7]

Some of the complications observed in patients with sclerosis and lupus erythematosus are polyserositis, pancreatitis, a vascular bone necrosis, arthritis, lung hypertension and renal failure. It is really important to distinguish between scleroderma renal crisis and lupus nephritis due to the different treatment that they have. Those patients with pulmonary hypertension can be benefited from the use of vasodilators. The new biological therapies seems to be solution to avoid the usage of high dosis of corticoids, especially in patients with severe kidney complications. But in the absence of reported clinical trials a conservative approach is recommended. [8]

\section{REFERENCES}

[1] Foocharoen C. Clinical characteristic of scleroderma overlap syndrome: comparisons with pure scleroderma. Int J Rheum Dis. 2016 ; 19(9:913-23

[2] Pasucci A. Lupus erythematosus and localized scleroderma coexistent at the same sites: a rare presentation of overlap syndrome of connective-tissue diseases. Cutis. 2016; 97(5):359-63

[3] Balbir-Gurman A. Scleroderma Overlap Syndorme. Isr Med Assoc J. 2011; 13(1):14-20.

[4] Valencia-Caballero V. Síndrome de superposición en esclerodermia: a propósito de un caso. Acta Med Peruana 2009;26:2

[5] Lidia Rudnicka. Atlas of Trichoscopy. Dermoscopy in hair and scalp disease.

[6] Tosti A. Follicular Red Dots. A Novel Dermoscopic Pattern Observed in Scalp Discoid Lupus Erythematosus Arch Dermal. 2009;145(12):1406-1409.

[7] Wells AU. High-resolution computed tomography and scleroderma lung disease. Rheumatology (Oxford). 2008;59-61

[8] Laccarino L, Gatto M, Bettio S, Caso F, Rampudda M, Zen M, Ghirardello A, Punzi L, Doria A. Overlap connective tissue disease syndromes. Autoimmun Rev. 2013 ;12(3):36373

Citation: Rosa Gimenez-Garcia, Donna Zhan, Diego Gonzalez-Gonzalez, Daniel Colinas-Reyero. Chronic Lupus Erythematosus and Systemic Scleroderma Coexistent at the Same Patient. ARC Journal of Dermatology. 2018; 3(1):4-5. doi:dx.doi.org/10.20431/2456-0022.0301002.

Copyright: (1) 2018 Authors. This is an open-access article distributed under the terms of the Creative Commons Attribution License, which permits unrestricted use, distribution, and reproduction in any medium, provided the original author and source are credited. 